\title{
A Scale Adaptive Hierarchical Clustered Routing Protocol for WSNs
}

\author{
Lipeng $\mathrm{Lu}^{1, a}$, Jiugen $\mathrm{Shi}^{2, b}$, and Huiliang $\mathrm{Xu}^{3, c}$ \\ $12{ }^{3}$ School of Computer and Information, Hefei University of Technology, Hefei, Anhui 230009, \\ China \\ ${ }^{a}$ Ilplyj@mail.hfut.edu.cn, ${ }^{b}$ shijg@hfut.edu.cn, ' xuhuiliang@mail.hfut.edu.cn
}

Keywords: WSNs, Routing protocol, Stable performance, Scale adaptive

\begin{abstract}
Due to technique advances, wireless sensor networks (WSNs) are widely used in both military and civil scenarios. A vast amount of data is transmitted to the base station through resource-restrained nodes, which are powered by batteries with limited energy. An energy saving routing protocol is fundamental for network lifetime prolongation. In this paper, we propose TECH (Tree-based Energy-balance Clustering Hierarchy), a scale adaptive hierarchical clustered routing protocol for WSNs. TECH starts the topology construction process from the base station to the far nodes, and then balance the load using the cluster heads within the same level. The in-cluster dynamic data gathering method is used for preventing the cluster heads from dying quickly and reducing the redundant data from transmission. We finally conduct extensive experiments by simulations to evaluate the performance of the proposed protocol. The simulation results show that the proposed protocol outperforms existing protocol in network lifetime prolongation. Furthermore, the protocol can be used in both small and large scale networks, and its performance is stable.
\end{abstract}

\section{Introduction}

Wireless sensor network is a distributed system, which usually consists of a base station and numerous of sensors. Sensors monitor the environment and send their sensory data to the base station directly or through relay nodes. As a new information collect and process technology, WSNs are widely used. Due to the attribute of distributed system and small size of the sensor, it can only be powered by small batteries. And in many applications, to change or recharge battery is either impossible or very expensive. Meanwhile, in WSNs, the energy cost on communication is always far more than that on computation and sensing [1]. All the above factors present us a clear truth, develop an efficient route protocol is essential to prolong the network lifetime.

At present, energy saving routing protocol in wireless sensor networks can be roughly divided into two sets according to the optimization target.

- minimize the total energy cost.

- maximize the network lifetime.

The former considers the energy saving problem in the macroscopic aspect. It combines the traditional cable network with radio radiation model and tries to reduce the redundant data needed to be sent and energy cost per byte, e.g. SPIN [2] and Directed Diffusion [3]. The later solves the problem considering both the macroscopic and microscopic aspects. On the basis of reducing energy consumption, seeking the load balance of the network, and preventing the partial nodes running out of energy, it dedicates to prolong the lifetime of the network, e.g. LEACH [4], FA [5], ENCAST [6], and DEER [7].

In this particular paper, we discuss wireless sensor networks where:

- Only one base station is placed in the center of the network.

- All nodes in the network are homogeneous and energy-constrained with the same capacity.

- All nodes send the sensory data to base station periodically.

To prolong the network lifetime, we develop a novel routing protocol, TECH, a scale adaptive hierarchical clustered routing protocol that minimizes energy dissipation in wireless sensor networks and the total energy consumption. The Key features of TECH are: 
- Hierarchical and clustered network topology.

- In-cluster data compression.

- Balanced network load.

- Aggregation of death nodes.

Related Work. In recent years, the routing protocols have been extensively studied in WSNs. LEACH [4] is the first clustered routing protocol. Its feature of randomized rotation of choosing cluster head can balance the network load effectively. But when applied to large scale network, the performance degradation is very severe. And then plenty of researches have been done to optimize the LEACH, e.g. [8, 9, 10]. Tree topologies are widely used in WSNs in order to route data to the sink. [11] considered the shortest path routing tree in WSNs under different metrics. [12] proposed a routing protocol with evenly distributed cluster head position. And it was proposed to solve fixed physical location for airfield lighting in airport lamp in which some lamps location is irregular. As mentioned, the routing protocol is essential to prolong network lifework. LEACH is not well suited in large scale network. And [12] is designed to be used in a particular scene. [16] proposed another cluster-based protocol, HEED. The cluster head needs to communicate with the base station directly. The energy consumption is severe on that.

Contributions. In this paper we consider the routing protocol in WSNs to maximize the network lifetime. The main contributions are as followers.

- We first introduce the work done by others and compare the works. And then we introduce a realistic cost model to evaluate the proposed routing protocol.

- We then devise a novel routing protocol, which is not only cluster-based but also hierarchical.

- We finally conduct extensive experiments via simulation. The experimental results show that the proposed routing protocol outperforms LEACH in terms of total energy consumption and the network lifework. Moreover, the proposed routing protocol can be used in both small and large scale network and its performance is stable.

The rest of the paper is organized as follows. Section 2 introduces the system model and problem definition. Section 3 proposes a scale adaptive hierarchical clustered routing protocol for WSNs. To evaluate the performance of the proposed routing protocol, extensive simulations are conducted in section 4 . The conclusions are given in section 5.

\section{Preliminaries}

System Model. We consider a sensor network G(V,E), where V is the set of sensors and $E$ is the set of links, consisting of $\mathrm{N}$ stationary sensors $v_{1}, v_{2}, \ldots, v_{N}$, randomly deployed in a region of interest, and a base station $r$ with unlimited energy supply located at the center of the region. We further assume that the transmission ranges of all sensors are identical, and each sensor can communicate with the base station via one or multi-hop relays. Otherwise, the sensor is not in the network. In this paper, each sensor has a point $\mathrm{p}$ and p.sid as well as p.val is represented by 4 bytes. Thus, a point $\mathrm{p}$ is represented by 8 bytes in total. To transmit a message containing $\mathrm{k}$ bytes of data from a sensor to one of its neighboring sensor with the distance $d$, the amount of transmission energy consumed at the sender is (1) [4] and the amount of reception energy consumed at the receiver is (2) [4]. We also assume that the sensing and computation energy consumption on sensors are not taken into account, because in practice they are several orders of magnitude less than that of wireless communication energy consumption. For example, the authors in $[13,14]$ claimed that the transmission of 1-bit data consumes as much as executing 1,000 CPU instructions. Therefore, unless otherwise specified, we only compare the communication energy consumption of different protocols in the later performance evaluation.

$$
\begin{aligned}
& E_{T x}(\mathrm{k}, \mathrm{d})=E_{\text {elec }} * k+\varepsilon_{\text {fs }} * k * \mathrm{~d}^{2} . \\
& E_{R x}(k)=E_{\text {elec }} * k .
\end{aligned}
$$


Problem Definition. Given a wireless sensor network, assume that $P\left(v_{i}\right)$ is the set of points generated at the sensor $v_{i}$, then $P=\cup_{i=1}^{N} P\left(\mathrm{v}_{i}\right)$ is the point set of the whole sensor network. Every sensor has to send the data to the base station through one or multi relays. So the base station can collect all the data generated by the network and monitor the region. The routing protocol problem is defined as follows. Since the sensors generate sensory data about the monitoring environment periodically, the problem is to propose a routing solution for every sensor to send its sensory data to the base station. Meanwhile, the total energy consumption and the maximum energy consumption among the sensors for routing their data to the base station are minimized with the ultimate aim to maximize the network lifetime. The network lifetime is referred to as the time when the first sensor in the network exhausts its energy [15].

The following important statement is the cornerstone of the network discussed in the rest of the paper.

Definition 1: We called the network is connected if and only if every two nodes in G(V,E) are connected. That is to say the network is randomly and homogeneously deployed, so every sensor can route its data to the base station. And the network we discussed in the rest of this paper is connected.

\section{TECH Protocol Details}

In this section, we describe the proposed protocol in details. The operation of TECH is broken into different stages. Each stage is the basis of the next stage. After all stage being processed in turn, the route for every node in the network will be established.

Establish Network Topology Roughly. In this stage, the protocol establishes a rough network topology according to the physical location of all sensors deployed in the network. The base station starts the topology establishing process, and then the process goes round by round till it reaches the nodes far from the base station. In other words, the process won't stop until it reaches the boundary of the network.

The detailed steps in this stage are displayed as follows. Firstly, the base station advertises the "build-network-message" to the network. And any node which receives the message will reply. Those nodes, which reply to the base station, are called the level-one nodes. The base station is a level-zero node for simplicity. Then, the level-one nodes advertise the "build-network-message" one by one. At this step, only the next level nodes will reply to the message and those nodes, which reply to the message, are called level-two nodes. The previous level nodes or called the father nodes should never reply to the message. This step goes round by round. Finally, no new next level nodes appear.

After the stage ends, a rough network topology is established. The nodes in the network are divided into different levels. Meanwhile, the nodes in the same level are divided into different clusters. But the topology is far from optimal. And the nodes in the present level may have multi fathers in the previous level. The distance between them are in the communication range. The protocol will choose the nearest father for the sake of saving energy on communication. This decision is based on the received signal strength (RSSI) of the advertisement. After this greedy optimization measure, the direct communication costs between the cluster head and the nodes in the cluster are much lower.

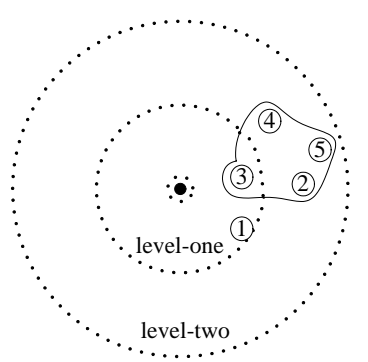

Fig. 1. Network topology in stage one.

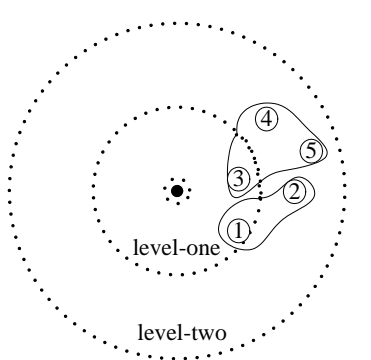

Fig. 2. Network topology in stage two. 
Fig. 1 depicts a rough network topology. The black dot represents the base station. The network is divided into two levels as shown in Fig. 1. Node 1 and 3 are the level-one nodes. Node 2, 4 and 5 are the level-two nodes. Node 3 is a cluster head with three members, node 2, 4 and 5 . Node 1 is a cluster with no cluster member. And node 2 is in the communication range of both cluster heads 1 and 3. Obviously, cluster head 3 will exhaust its energy much quicker than cluster head 1.

Balance the Cluster Size. A rough network topology, including all sensors if the network is connected (definition 1), is established. But the topology is not optimal. In current stage, the protocol is going to optimize the topology by balancing the cluster size.

As we discussed in the above stage, the network topology is hierarchical. And we declare that all nodes in the same cluster are not only physically close but also in the same level and the cluster head is in the previous level. So the cluster size can be balanced if all or partial nodes in a cluster are in the communication range of multi nodes in the previous level. The nodes like that have multi potential cluster heads.

Definition 2: The nodes, which have more than one potential cluster heads, are called "to-be-optimized-nodes". Those "to-be-optimized-nodes" are the key optimization objective in this stage of protocol.

This stage is processed round by round as well. The protocol runs from level one to the last level. In each level, those "to-be-optimized-nodes" (definition 2) will be picked up and switch to the cluster containing fewer nodes according to the cluster size registered in the cluster heads. So the network load will be divided evenly. This can prevent the cluster head of big cluster size from dying effectively.

Fig. 2 shows us a network topology after node 2 ("to-be-optimized-node") changes its cluster head. The load of cluster head 3 is balanced with the help of cluster head 1 .

Dynamic in-cluster Data Collecting. The traditional cluster-based protocol, like LEACH, uses the cluster heads to collect the data as long as possible. This leads to quick exhausting energy of the cluster heads. The routing protocol we proposed in this paper doesn't act like that.

The dynamic in-cluster data collecting process is described as follows. At first, the cluster head will collect the data in its cluster and fuse the data. After a few rounds, the cluster head sends "ask-remaining-energy-message" to the nodes in its cluster. And the nodes reply to the message along with its remaining energy. Then, the cluster head decide the node with the most remaining energy to collect and fuse the data. And the fused data will be sent to the cluster head eventually. The nodes in the same cluster bear the load together through this dynamic data collecting strategy.

The protocol we proposed only balance the load in cluster, not in the whole network, so there isn't much energy consumption via advertise messages and no energy consumed to dynamically reconstruct the network topology, like LEACH. In order to further reduce the energy consumption, this dynamic deciding work only happens after a period of steady transmission.

More Details. Through simulation, we find that the simulation results will be better if we place a little bit more nodes in the communication range of the base station. More nodes in the first level mean more potential branches to extend the network topology. This leads to smaller cluster size and less level. The system can determine, a priori, the optimal number of nodes to place near the base station. For our system parameters and topology, we find that $5 \%$ of nodes are optimal.

\section{Performance Evaluations}

In this section, we evaluate the performance of the proposed routing protocol via simulation. The simulations are conducted to study the network lifetime in terms of the following factors, number of rounds when $1 \%, 20 \%, 50 \%$, and $100 \%$ nodes die, various network dimensions $100 \mathrm{~m} \times 100 \mathrm{~m}$, $500 \mathrm{~m} \times 500 \mathrm{~m}, 1000 \mathrm{~m} \times 1000 \mathrm{~m}$ and we vary the radiation radius at $40 \mathrm{~m}, 70 \mathrm{~m}$, and $100 \mathrm{~m}$ to study how the protocol works with low to high coverage ranges. We also study the death nodes distribution. And at last, we evaluate the performance of the proposed protocol at different densities of nodes. The performance of algorithm LEACH will be used as the benchmark for comparison purpose.

Simulation Setting. We assume that the sensor network is randomly deployed to monitor a 
region of interest, in which $\mathrm{N}$ sensors are deployed by MATLAB and the base station is located at the center of the region. Assume that all the sensors have the same transmission ranges $(40 \mathrm{~m}, 70 \mathrm{~m}$, and $100 \mathrm{~m}$ in this paper). As mentioned in section 2, we use the same radio model as LEACH. The initial energy at each sensor is 0.5J. The energy consumptions of transmitting and receiving one byte are $E_{T x}=50 n J$ and $E_{R x}=50 n J$, respectively.

Table 1. Simulation parameters.

\begin{tabular}{|l|l|l|}
\hline Type & Parameter & Value \\
\hline Network & Network size & $100(\mathrm{~m}) \times 100(\mathrm{~m}), 500(\mathrm{~m}) \times \mathrm{m} 00(\mathrm{~m}), 1000(\mathrm{~m}) \times 1000(\mathrm{~m})$ \\
& Base station & At the center of the network \\
& Initial energy & $0.5(\mathrm{~J} / \mathrm{battery})$ \\
\hline Application & Cluster radius & $40(\mathrm{~m}), 70(\mathrm{~m}), 100(\mathrm{~m})$ \\
& Data packet size & 3975 bytes \\
& Broadcast packet size & 25 bytes \\
& Packet header size & 25 bytes \\
\hline Radio model & $\mathrm{E}_{\mathrm{Tx}}=\mathrm{E}_{\mathrm{Rx}}=\mathrm{E}_{\text {elec }}$ & $50(\mathrm{~nJ} / \mathrm{bit})$ \\
& $\varepsilon_{\mathrm{fs}}$ & $10\left(\mathrm{pJ} / \mathrm{bit} / \mathrm{m}^{2}\right)$ \\
& $\varepsilon_{\mathrm{mp}}$ & $0.0013\left(\mathrm{pJ} / \mathrm{bit} / \mathrm{m}^{4}\right)$ \\
& $\mathrm{E}_{\mathrm{fusion}}$ & $5(\mathrm{~nJ} / \mathrm{bit} / \mathrm{signal})$ \\
\hline
\end{tabular}

Impact of Network Scale on Performance of Protocol. In this subsection, we evaluate the performance of TECH and LEACH in different network scales, assuming the radiation radius is $70 \mathrm{~m}$ and $D_{n d}=1 / 200$. The following definition is an important parameter to evaluate the performance of the protocol.

Definition 3: We define the nodes density of the network. The nodes density is calculated by the following formulation. $D_{n d}$ represents the node density of the network. $\mathrm{N}$ is the number of nodes in the network. And $\mathrm{S}$ is the area of the monitoring region.

$$
D_{n d}=N / S \text {. }
$$

Fig. 3 shows the comparison of the network lifetime in various network scales. TECH outperforms LEACH in different network scales. The performance of both protocols degrades with network expansion. But LEACH degrades more severe. Because the cluster heads communicate directly with the base station using LEACH. And with the network expansion the distance between cluster heads and the base station enlarges. So much more energy is consumed on communication. On the contrary, TECH will extend the network topology adding more levels. And the network lifetime remain almost unchanged in fig. 3(b) and fig. 3(c).

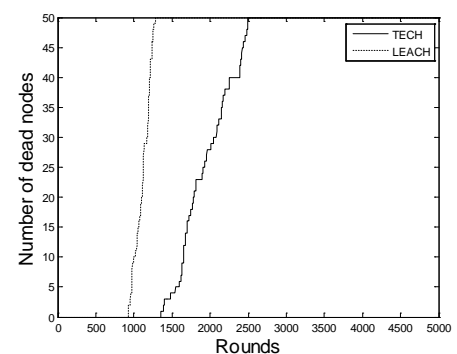

(a) $\mathrm{S}=100 \mathrm{~m} \times 100 \mathrm{~m}$.

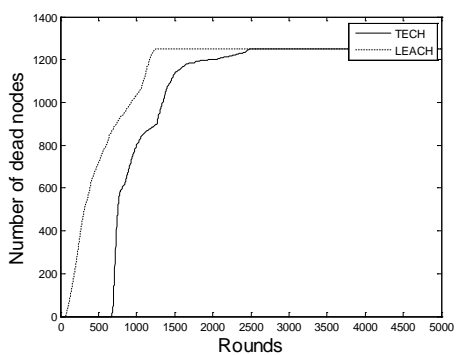

(b) $\mathrm{S}=500 \mathrm{~m} \times 500 \mathrm{~m}$.

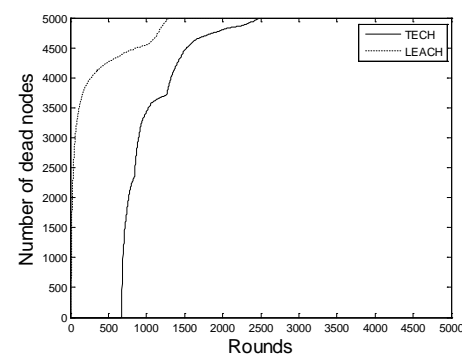

(c) $\mathrm{S}=1000 \mathrm{~m} \times 1000 \mathrm{~m}$.

Fig. 3. Comparison of TECH and LEACH in terms of network lifetime in various network scales.

Impact of Radiation Radius on Performance of Protocol. In this subsection, we investigate the impact of various radiation radiuses on the performance of the proposed protocol, assuming the network scale is $500 \mathrm{~m} \times 500 \mathrm{~m}$ and $D_{n d}=1 / 200$.

Fig. 4 plots the curves of the comparison of TECH and LEACH in terms of network lifetime 
when radiation radius $=40 \mathrm{~m}, 70 \mathrm{~m}$ and $100 \mathrm{~m}$, respectively. As shown in Fig. 4 , TECH outperforms LEACH in all radiation radiuses. The rounds of first dead node coming up are 753, 666, 571 for TECH and 61, 61, 64 for LEACH. The performance of both protocols degrades as the radiation radius increases because more energy consumed on in-cluster data gathering.

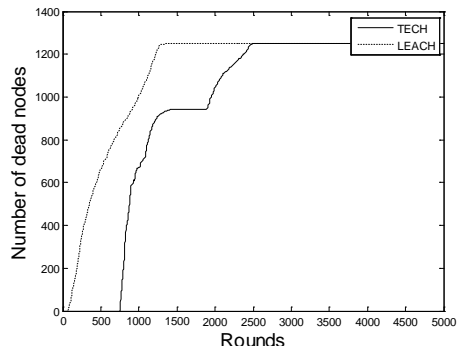

(a) Radius $=40 \mathrm{~m}$.

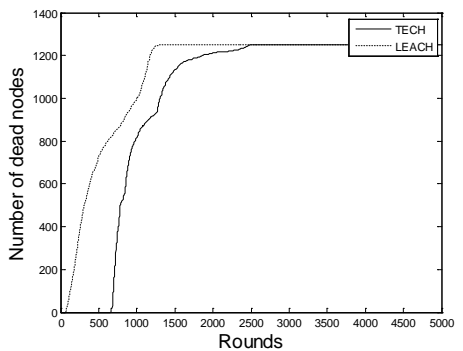

(b) Radius $=70 \mathrm{~m}$.

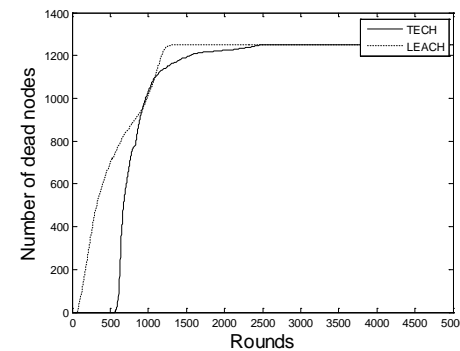

(c) Radius $=100 \mathrm{~m}$.

Fig.4. Comparison of TECH and LEACH in terms of network lifetime in various radiation radiuses.

Impact of Node Density on Performance of Protocol. In this subsection, we compare the performance of TECH and LEACH under different node densities, assuming the network scale is $300 \mathrm{~m} \times 300 \mathrm{~m}$, radiation radius is $40 \mathrm{~m}$ and $D_{n d}=1 / 360,1 / 90$ and $1 / 30$.

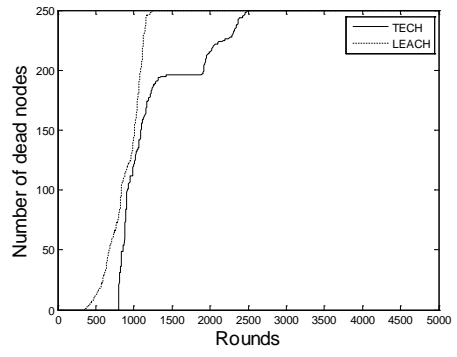

(a) $\mathrm{N}=250$.

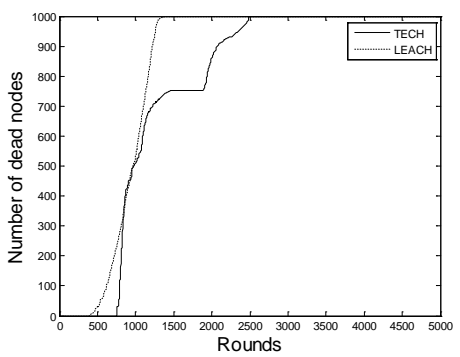

(b) $\mathrm{N}=1000$.

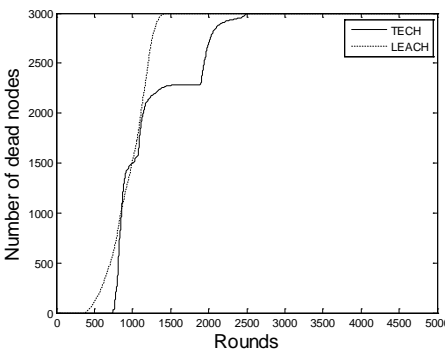

(c) $\mathrm{N}=3000$.

Fig. 5. Comparison of TECH and LEACH in terms of network lifetime in different node densities.

Fig. 5 shows us the simulation results of network lifetime for TECH and LEACH under various node densities. TECH outperforms LEACH. The combination of first dead node and last dead node in rounds are shown below. $(803,2492)$, $(352,1244)$ for TECH and LEACH from fig. 5(a), respectively. (750, 2499), (393, 1390) for TECH and LEACH from fig. 5(b). (745, 2501), (388, 1550) for TECH and LEACH from fig. 5(c). The performance of TECH remains changeless, and that of LEACH improves a little bit.

Comparison of Various Percentage of Node Death. In this subsection, we run the simulations to determine the number of rounds of communication when $1 \%, 20 \%, 50 \%, 100 \%$ of the nodes die using TECH and LEACH, assuming the network scale is $500 \mathrm{~m} \times 500 \mathrm{~m}, D_{n d}=1 / 200$ and radiation radius is $70 \mathrm{~m}$. Once a node dies it is considered dead for the rest of the simulation.

Table 2. The average number of rounds when $1 \%, 20 \%, 50 \%$ and $100 \%$ nodes die.

\begin{tabular}{|c|c|c|c|c|c|c|c|c|c|}
\hline \multirow{2}{*}{\multicolumn{2}{|c|}{$\begin{array}{l}\text { Protocol } \\
\text { Percentage }\end{array}$}} & \multicolumn{4}{|c|}{ TECH } & \multicolumn{4}{|c|}{ LEACH } \\
\hline & & $1 \%$ & $20 \%$ & $50 \%$ & $100 \%$ & $1 \%$ & $20 \%$ & $50 \%$ & $100 \%$ \\
\hline \multirow[t]{10}{*}{ Round } & 1 & 689 & 722 & 852 & 2495 & 78 & 203 & 449 & 1383 \\
\hline & 2 & 687 & 720 & 856 & 2487 & 76 & 197 & 417 & 1293 \\
\hline & 3 & 673 & 720 & 864 & 2483 & 83 & 211 & 423 & 1425 \\
\hline & 4 & 687 & 718 & 841 & 2491 & 80 & 204 & 428 & 1343 \\
\hline & 5 & 684 & 717 & 844 & 2493 & 75 & 204 & 430 & 1317 \\
\hline & 6 & 686 & 726 & 845 & 2434 & 87 & 210 & 456 & 1348 \\
\hline & 7 & 684 & 720 & 859 & 2499 & 76 & 201 & 428 & 1359 \\
\hline & 8 & 680 & 726 & 868 & 2488 & 83 & 207 & 453 & 1282 \\
\hline & 9 & 680 & 722 & 851 & 2500 & 78 & 201 & 452 & 1313 \\
\hline & 10 & 682 & 716 & 851 & 2492 & 80 & 201 & 423 & 1300 \\
\hline \multicolumn{2}{|c|}{ Average } & 683 & 721 & 853 & 2486 & 80 & 204 & 436 & 1336 \\
\hline
\end{tabular}


Fig. 6 shows the number of rounds until 1\%, 20\%, 50\% and 100\% nodes die. TECH is approximately $2 \mathrm{x}$ better than LEACH in all cases for a $500 \mathrm{~m}$ x 500m network.

Death Nodes Distribution. In this subsection, we discuss the problem of the death nodes distribution of TECH, assuming the network scale is $200 \mathrm{~m} \times 200 \mathrm{~m}$, radiation radius is $40 \mathrm{~m}$ and $\mathrm{N}=$ 100 .

Fig. 7 may display us a promising attribute of the proposed protocol. The nodes in the same cluster die together. The symbol $\left(^{*}\right)$ represents a node. The symbols on the same height in fig. 7 are the nodes in the same cluster. Some symbols on the same height stick tightly together and the other are close to each other with a little space in between. The physical meaning of this attribute is that the remaining energy of nodes in the same cluster is approximately the same.

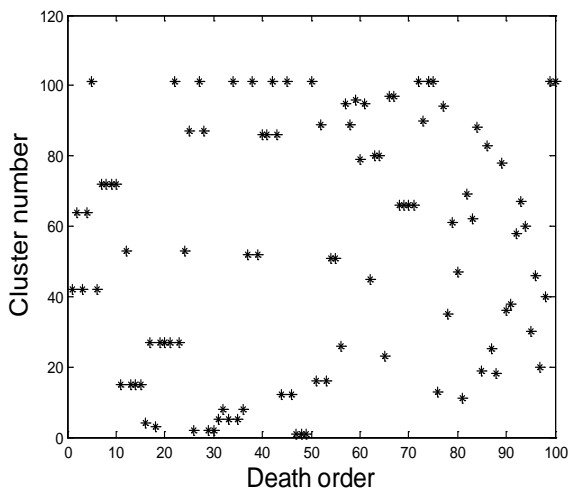

Fig. 7. Death node distribution.

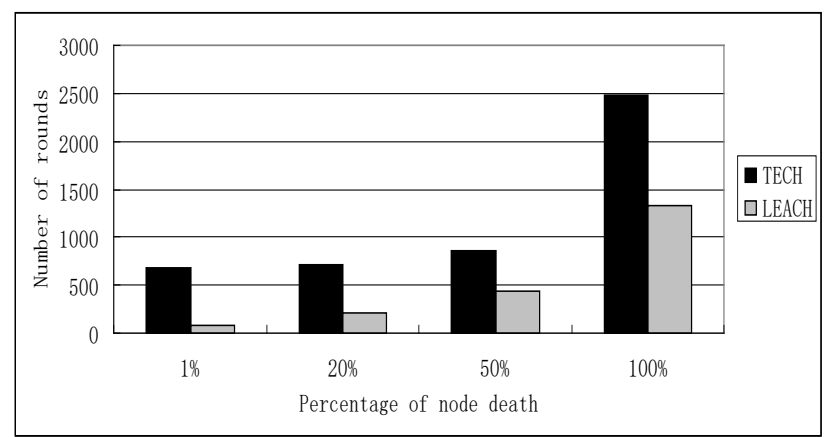

Fig. 6. Performance results for TECH versus LEACH.

\section{Conclusions}

Routing protocol is essential in terms of saving energy in wireless sensor networks. In this paper, we propose a novel routing protocol, TECH, a scale adaptive hierarchical clustered routing protocol, which is near optimal for solving data-gathering problem in wireless sensor networks. And we conduct impeccable experiments via simulation. The simulation results show us that TECH outperforms LEACH in various network scales and under different radiation radiuses. Distributing the energy load among the nodes increases the lifetime and quality of the network. Our simulations demonstrate that TECH performs better than LEACH by about $2 \mathrm{x}$ when 1\%, 20\%, 50\% and 100\% of nodes die. And the death nodes distribution curve shows us a promising future when TECH being used in reality. TECH shows an even further improvement as the scale of the network increases.

\section{Acknowledgement}

This project is supported by the National Key Scientific Instrument and Equipment Development Project (2013YQ030595).

\section{References}

[1] D. Niculescu. Communication Paradigms for Sensor Networks. IEEE Communications Magazine. 2005, 116-122.

[2] Kulik J, Heinzelman WR, and Balakrish H, Negotiation Based Protocols for Disseminating Information in Wireless Sensor Networks. Wireless Networks. August 2002, 169-185.

[3] Intanagonwiwat C, Govindan R, Estrin D, Heidemann J. Directed Diffusion for Wireless Sensor Networking. IEEE/ACM Trans on Networking. 2003, 2-16.

[4] W. R. Heinzelman, A. Chandrakasan, H. Balakrishnan. Energy-Efficient Communication Protocol for Wireless Microsensor Networks. In Proc. Proceedings of the 33rd Annual Hawaii International Conference on System Sciences. January 2000, 3005-3014. 
[5] JH Chang, L Tassiulas. Maximum Lifetime Routing in Wireless Sensor Networks. IEEE/ACM Trans on Networking. 2004, 609-619.

[6] S. Zou, I. Nikolaidis, J. J. Harms. ENCAST: Energy critical Node Aware Spanning Tree for Sensor Networks. In Proc. 3rd Annual Communication Networks and Services Research Conference. 2005, 249-254.

[7] Y Wang, H Wu, R Nelavelli, NF Tzeng. Balance-Based Energy-Efficient Communication Protocols for Wireless Sensor Networks. In Proc. 26th IEEE International Conference on Distributed Computing Systems Workshops. 2006, 85-85.

[8] Deng Zhixiang, Qi Bensheng. Three-Layered Routing Protocol for WSN Based on LEACH Algorithm. In Proc. IET Conference on Wireless, Mobile and Sensor Networks. Dec. 2007, 72-75.

[9] Wei Zhang, Liang Zhao. Analysis and Research of Improved LEACH Routing Protocol for Pressure Sensor Based on WSN. In Proc. International Conference on Cyberspace Technology. Nov. 2014, 1-6.

[10] Sujee R., Kannammal K. E.. Behavior of LEACH Protocol in Heterogeneous and Homogeneous Environment. 2015 International Conference on Computer Communication and Informatics. Jan. 2015, 1-8.

[11] Walid Bechkit, Mouloud Koudil, Yacine Challal, Abdelmadjid Bouabdallah, Brahim Souici, Karima Benatchba. A new Weighted Shortest Path Tree for Convergecast Traffic Routing in WSN. In Proc. 2012 IEEE Symposium on Computers and Communications. July 2012, 187-192.

[12] Bing-Yuan Wang, Xin-Qing Zhang, Kun Tian, Mei Gao. An Efficient WSN Routing Protocol on Airfield Lighting Monitoring System. In Proc. 2015 Fifth International Conference on Instrumentation and Measurement, Computer, Communication and Control. Sept. 2015, 1701-1705.

[13] S. Madden, M. J. Franklin, J. M. Helleratein, W. Hong. The Design of an Acquisitional Query Processor for Sensor Networks. In Proc. ACM SIGMOD. 2003, 491-502.

[14] G. J. Pottie, W. J. Kaiser. Wireless Integrated Network Sensors. Communication of ACM. Vol.43 No.5, pp.51-58, 2000.

[15] Jae-Hwan Chang, L. Tassiulas. Energy Conserving Routing in Wireless Ad-hoc Networks. In Proc. INFOCOM 2000. Mar. 2000, 22-31.

[16] Ossama Younis, Sonia Fahmy. HEED: A Hybrid, Energy-Efficient, Distributed Clustering Approach for Ad Hoc Sensor Networks. IEEE Transactions on Mobile Computing. 2004, 366-379. 